\title{
Magnetic Properties of Iron Core Compacted Powders Coated with Magnetic Insulative Film*
}

\author{
Junghwan Hwang, Takeshi Hattori and Yuji Kaneko \\ Toyota Central R\&D Labs. Inc., Nagakute 480-1192, Japan
}

To improve the permeability and magnetic flux density of a powder magnetic core, we examined iron core compacted powders coated with spinel ferrite particles. Their permeability and magnetic flux density are higher than those of iron core compacted powders coated with nonmagnetic insulative film having equal resistivity: $1.4 \mathrm{~T}$ magnetic flux density $B_{2 \mathrm{k}}, 7.79 \mathrm{Mg} / \mathrm{m}^{3}$ green density, and $70 \mu \Omega \cdot \mathrm{m}$ resistivity were achieved. [doi:10.2320/matertrans.Y-M2015836]

(Received September 15, 2015; Accepted October 31, 2015; Published December 18, 2015)

Keywords: magnetic insulative film, spine-type ferrite, powder magnetic core, permeability, magnetic flux density

\section{Introduction}

Powder magnetic cores have several benefits. (1) They can be used to design a three-dimensional magnetic circuit from isotropic magnetism characteristics. (2) They also have low eddy current loss at high-frequency areas because of their high resistivity: the powder magnetic core comprises compacted powders coated with insulative films. Those benefits can improve applications to choke coils for electronic equipment, ${ }^{1-3)}$ injection valves ${ }^{4)}$ and motors. ${ }^{5,6)}$

However, present powder magnetic cores present several difficulties: their magnetic flux density and strength are lower than those of electrical steel sheets. Normally, a powder magnetic core comprises particles of $100 \mu \mathrm{m}$ diameter with surfaces coated with nonmagnetic insulative films such as silicone resins and phosphates. Therefore, it is difficult to create a magnetic field and achieve high magnetic flux density because the demagnetizing field within the powders produces magnetic resistance. In contrast, a magnetic powder core with a magnetic insulative film is magnetized easily. Such a core offers improved permeability because of relaxed magnetic resistance of the powders.

Among earlier studies of applications to spinel-type ferrite, which has high resistivity to insulative films, some studies found improved permeability of the powder magnetic core in areas with frequencies higher than 100 $\mathrm{kHz}$. Abe $\mathrm{As}^{7,8)}$ developed soft magnetic materials that operate at frequencies higher than $100 \mathrm{kHz}$ and developed a particle surface using ferrite plating coated with spinel-type ferrite film having thickness of several dozen micrometers. ${ }^{9,10)}$ That film uses characteristics of high permeability and high electrical resistivity of spinel-type ferrite $\mathrm{MFe}_{2} \mathrm{O}_{4}(\mathrm{M}=\mathrm{Ni}$, $\mathrm{Zn}, \mathrm{Fe}, \mathrm{Mn})$. Hirota ${ }^{11,12)}$ used compaction and pulse sintering to manufacture composites with $6 \mathrm{Mg} / \mathrm{m}^{3}$ density. Then he used ferrite-powder-coated powders that had been produced using mechano-fusion, which can impart great shear stress.

Nevertheless, because these studies used a high-frequency domain of more than $100 \mathrm{kHz}$, the fine particle surface required application of a thick film. Such thick films reduced

*This Paper was Originally Published in Japanese in Japan Soc. Powder Powder Metall. 61 (2014) 556-562. the saturation magnetization $B_{\mathrm{s}}$ to less than $1.5 \mathrm{~T}$. Therefore, this study was conducted to achieve higher magnetic flux density and higher permeability of the powder magnetic core simultaneously by combining high-density compaction and magnetism of the insulative film for a domain in which a frequency of several kilohertz is used.

For this study, the magnetization in the insulative film was assessed by determining the influence of the saturation magnetization of the magnetic insulative film and by determining the influence of the iron powder particle size on the permeability (herein, magnetic flux density $B_{2 \mathrm{k}}(H=$ $2000 \mathrm{~A} / \mathrm{m}$ ) was examined instead of the permeability). ${ }^{13)}$ Then, iron spherical powders and spinel-type ferrite powders were mixed. Thereby, the pure iron powder particle surfaces were coated with ferrite. Compaction was used for the coated powders. Then the characteristics were evaluated for the magnetic powder core. After the evaluation, a comparison was made between experimental values and calculated values of the effect of the magnetic insulative films. Results demonstrated the possibility of high saturation magnetization for a powder magnetic core according to the magnetism of insulative films.

\section{Experiment Methods}

\subsection{Calculation of magnetic flux density}

Calculation of the magnetic flux density using the model reported by Kawase was done to ascertain the magnetic field strength. ${ }^{14)}$ The analyzed model and the formula used to calculate the magnetic flux density $B$ for the powder magnetic core are presented in Fig. 1. The magnetic flux density generated in one powder particle is determined by the powder diameter $\left(l_{2}\right)$ and insulative film thickness $(g)$, from which the gap is calculated. The values of parameters used for calculation are presented below. The magnetic reluctivity of the iron powder $(v)$ of $1.45 \mathrm{~T}$ was used, which was the value of magnetic flux density $B_{2 \mathrm{k}}$ evaluated using a non-coated ring test piece of iron powder compacted at $1568 \mathrm{MPa}$. Magnetic reluctivity $\left(v_{0}\right)$ was a different value for each type of insulative film.

First, for a nonmagnetic film, the magnetic reluctivity $\left(v_{0}\right)$ in vacuum was used. Then, for a magnetic insulative film, the magnetic reluctivity $v_{0}$ was used for magnetic resistivity for 


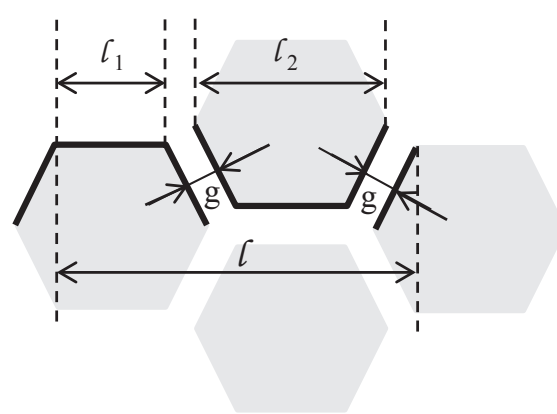

$$
\begin{aligned}
& \int H \mathrm{~d} \digamma=v B\left(\complement_{1}+\varsigma_{2}\right)+2 v_{0} B \frac{2}{\sqrt{3}} \mathrm{~g}=H \digamma \\
& H: \text { magnetic field intensity } \\
& B: \text { magnetic flux density } \\
& v: \text { magnetic reluctivity in magnetic substance } \\
& v_{0}: \text { magnetic reluctivity in vacuum } \\
& \complement: \text { unit length of powder magnetic core } \\
& \complement_{2}: \text { powder diameter }\left(\complement_{1}=\varsigma_{2} / 2\right) \\
& \mathrm{g}: \text { insulative film thickness }
\end{aligned}
$$

Fig. 1 Analyzed model and calculation formula of magnetic flux density $B$ for a powder magnetic core.

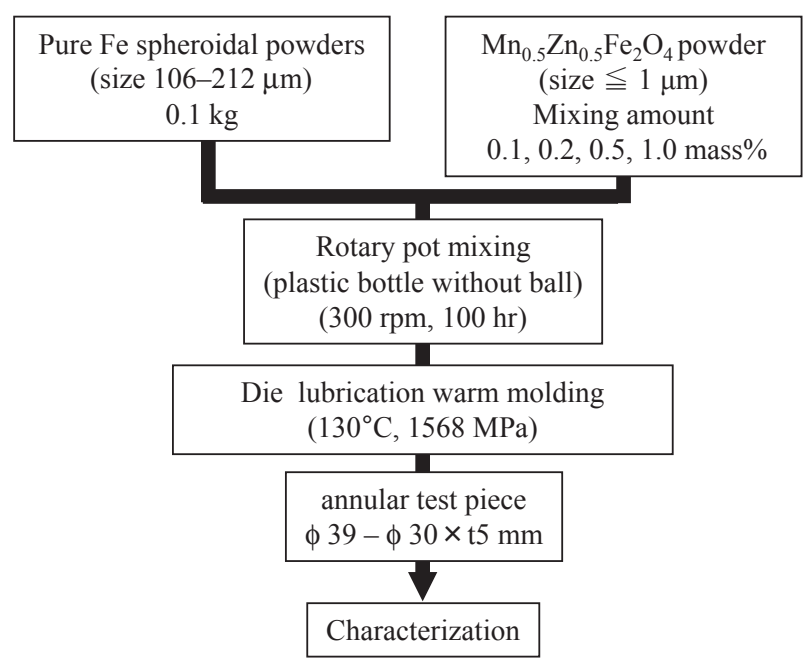

Fig. 2 Experimental procedure of powder magnetic core using magnetic insulative film.

spinel-type ferrite saturation magnetization on the premise that the spinel ferrite's saturation was $2000 \mathrm{~A} / \mathrm{m}$.

\subsection{Conditions of test piece manufacture and character- istic evaluation}

The test piece manufacturing procedure is presented in Fig. 2. Raw magnetic powders used $200 \mathrm{~g}$ of pure iron gas atomizing spherical powder with particle size distribution of $106-212 \mu \mathrm{m}$. The spinel-type ferrite powders used for coating onto the raw magnetic powder surface were $\mathrm{Mn}_{0.5} \mathrm{Zn}_{0.5} \mathrm{Fe}_{2} \mathrm{O}_{4}$ of less than $1 \mu \mathrm{m}$ particle diameter (KOJUNDO Chemical Laboratory Co., LTD.). After measurement, the saturation magnetization of the ferrite powder was $0.2 \mathrm{~T}$. Spinel-type ferrite powders were weighed as about 0.1-1.0 mass\% for raw magnetic powders after putting the powders with raw magnetic powders into a $500 \mathrm{ml}$ plastic bottle. Then the plastic bottle was rotated at $300 \mathrm{rpm}$ for $100 \mathrm{hr}$ to achieve thorough coating.

Powders coated with phosphate film, a nonmagnetic insulative film, were used as materials for comparison. First, $100 \mathrm{~g}$ of the same raw magnetic powders were put into a 100 $\mathrm{ml}$ beaker. Then phosphate solution ${ }^{15}$ ) (which was prepared for a molar ratio of $\mathrm{SrO}: \mathrm{H}_{3} \mathrm{BO}_{3}: \mathrm{H}_{3} \mathrm{PO}_{4}=1.5: 1.0: 4.0$ ) was added. Subsequently, those mixtures were put into a furnace to dry at $523 \mathrm{~K}$ after stirring with a spatula. Then the powder was dried for $30 \mathrm{~min}$ at room temperature and set up for decomposition. The test piece was annular: 39-

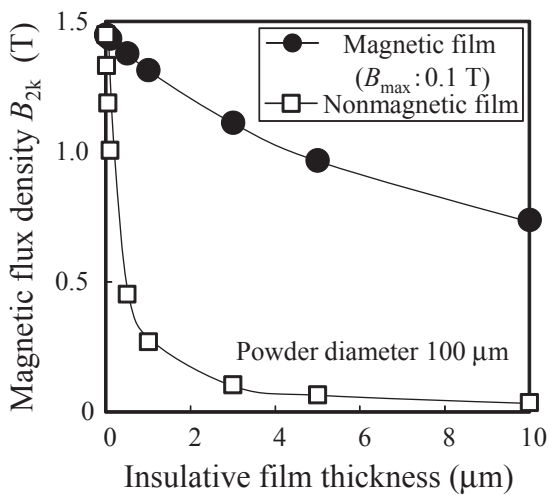

Fig. 3 Effect of magnetism of the insulative film.

$30 \times \mathrm{t} 5 \mathrm{~mm}$. Compaction was done using die lubrication warm molding ${ }^{16,17)}$ with lithium stearate. After heating and storage at $423 \mathrm{~K}$ die temperature, it was compacted at $1568 \mathrm{MPa}$. Neither internal lubricants nor resins were added for compaction. The green density, resistivity, and magnetic properties were evaluated thereafter. The green density was measured by dimension density from its weight and shape. The resistivity was measured using the four-terminal method with a micro-ohm meter (R6561; Advantest Corp.). For measurement of the static magnetic field properties, an annular test piece with 80 turns for detection and 240 turns for excitation of coating copper wire of $0.5 \mathrm{~mm}$ was used. The measuring equipment was operated using a direct current $J-H$ curve tracer (TRF-5; Toei Industry Co. Ltd.). $B_{X \mathrm{k}}$ denotes the magnetic flux density measured at $X \mathrm{kA} / \mathrm{m}$. Cross-sections of the surface of the coating powders and magnetic core were observed using scanning electron microscopy (SEM).

\section{Results}

\subsection{Calculated values of magnetic flux density $B_{2 k}$ depending on the insulative film thickness}

The calculated results for insulative film thickness and magnetic flux density $B_{2 \mathrm{k}}$ in nonmagnetic and magnetic insulative films with saturation magnetization of $0.1 \mathrm{~T}$ are presented in Fig. 3. The iron powder particle diameter was $100 \mu \mathrm{m}$. Results show that magnetic flux density $B_{2 \mathrm{k}}$ of the magnetic film having saturation magnetization of $0.1 \mathrm{~T}$ was greater than in the nonmagnetic film of equal film thickness. Moreover, improvement of the magnetic flux density of the 


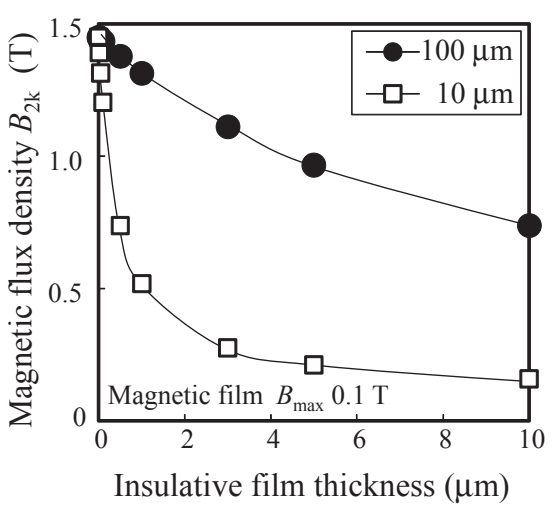

Fig. 4 Effect of particle diameter.

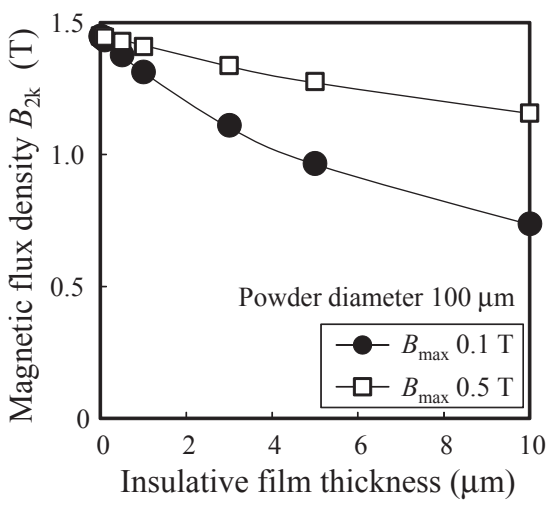

Fig. 5 Effect of $B_{\max }$ of the magnetic insulative film thickness on flux density.

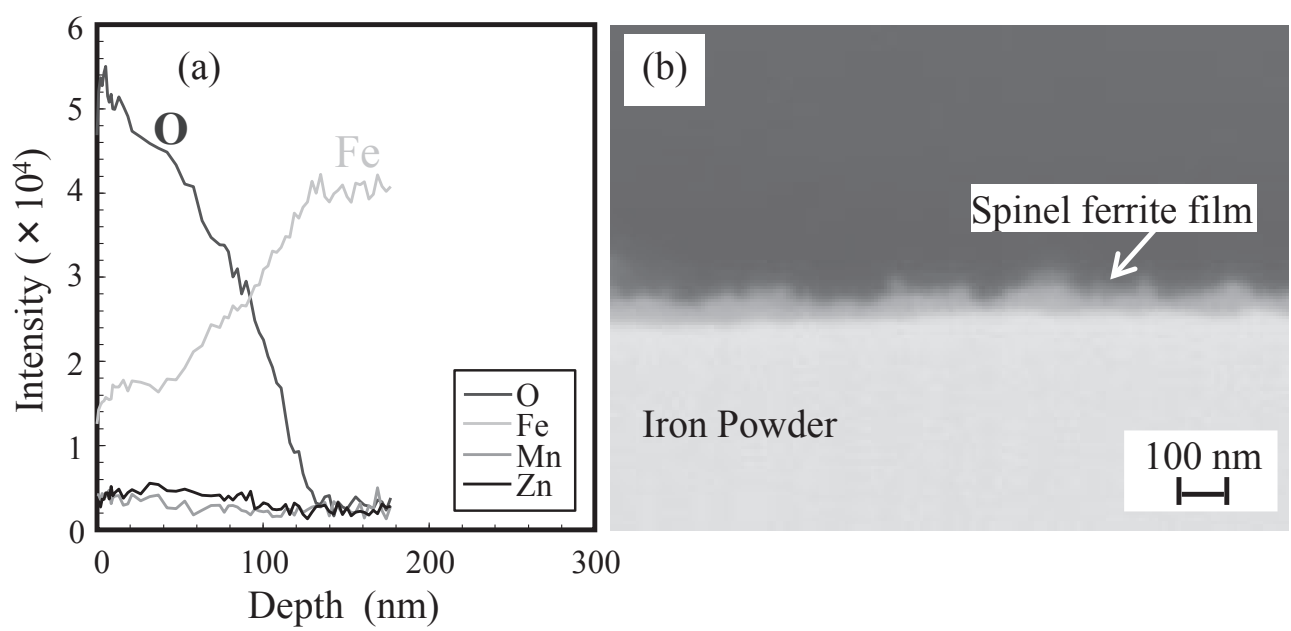

Fig. 6 Measurement of spinel-type ferrite film thickness: (a) analysis result of AES depth spectrum and (b) SEM image of powder crosssection.

powder magnetic core was achieved using a film with magnetism instead of a nonmagnetic film. The effect of the diameter of magnetic insulative film coating particles in relation to the film thickness and magnetic flux density $B_{2 \mathrm{k}}$ is presented in Fig. 4. When the film thickness was increased, the magnetic flux density $B_{2 \mathrm{k}}$ decreased. Then magnetic flux density $B_{2 \mathrm{k}}$ was increased so that the particle diameter became greater for equal film thickness.

In other words, results confirmed that magnetic flux density $B_{2 \mathrm{k}}$ was increased so that percentage of iron powders in compaction was increased. The saturation magnetization of the magnetic film and magnetic flux density $B_{2 \mathrm{k}}$, which changed according to the film thickness, are presented in Fig. 5. The iron powder particle diameter was $100 \mu \mathrm{m}$. Usage of a magnetic film with high saturation magnetization revealed that magnetic flux density $B_{2 \mathrm{k}}$ was improved, which presents benefits for achieving magnetic flux density.

\subsection{Characteristics of the spinel-type ferrite coating powder}

To confirm the calculation result described above, a ring test piece of compacted spinel-type ferrite coating iron powders was examined. First, the thickness of the insulative film produced from mixed amounts of spinel-type ferrite powders was measured to ascertain the relation of the magnetic insulative film thickness to several important properties: green density, permeability, magnetic flux density $B_{2 \mathrm{k}}$, and resistivity. The thickness was measured using Auger electron spectroscopy (AES). The distribution of the oxygen thick direction of the film was measured from the coating powder surface. The thickness that gave $50 \%$ of the surface oxygen strength was regarded as the ferrite film thickness. The measured value was the average value obtained from analyses of two powders, with measurements taken at two places for each powder. Results of the AES analysis and an SEM cross-section image of the spinel-type ferrite coating powders are presented in Fig. 6. The film thickness at oxygen strength of 26,000 that gives $50 \%$ of oxygen strength of 52,000 at the surface was approximately $100 \mathrm{~nm}$, as depicted in Fig. 6(a). The film thickness of $100 \mathrm{~nm}$ obtained by crosssection of the coating powder shown in Fig. 6(a) is depicted in Fig. 6(b). The relations of the mixing amount of the spineltype ferrite powders and the insulative film thickness measured using AES analysis are portrayed in Fig. 7. Results show that the thickness became approximately $50 \mathrm{~nm}$ for the mixed amount of 0.1 mass $\%$ and $200 \mathrm{~nm}$ of the mixing amount of 0.5 mass $\%$ of the spinel-type ferrite. Green density, resistivity, and magnetic flux density of $B_{2 \mathrm{k}}$ and $B_{20 \mathrm{k}}$ are presented in Fig. 8 according to the magnetic insulative film thickness. 


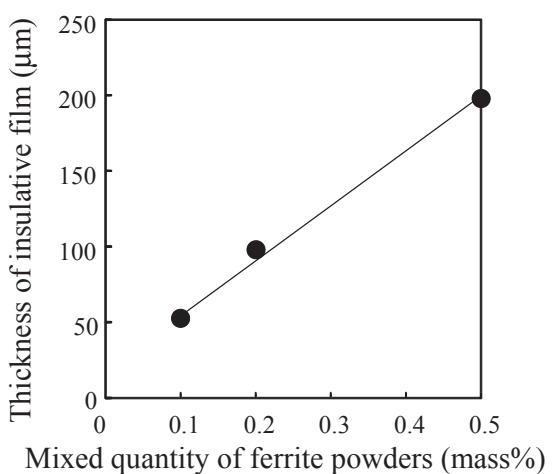

Fig. 7 Relation between addition quantity of spinel-type ferrite powders and film thickness $\left({ }^{*}\right.$ film thickness was analyzed using the AES depth spectrum).

As an insulative film becomes thicker, its resistivity increases along with the insulation characteristic improvement, but the green density and magnetic flux density of $B_{2 \mathrm{k}}$ and $B_{20 \mathrm{k}}$ decrease. Properties of the powder magnetic core were elucidated for a 50-nm-thick film of the coating powders: $70 \mu \Omega \cdot \mathrm{m}$ resistivity, $7.79 \mathrm{Mg} / \mathrm{m}^{3}$ green density, magnetic flux density $B_{2 \mathrm{k}}$ of $1.4 \mathrm{~T}$, magnetic flux density $B_{20 \mathrm{k}}$ of $1.96 \mathrm{~T}$. They were also elucidated for a 100 -nm-thick film: $100 \mu \Omega \cdot \mathrm{m}$ resistivity, $7.78 \mathrm{Mg} / \mathrm{m}^{3}$ green density, magnetic flux density $B_{2 \mathrm{k}}$ of $1.3 \mathrm{~T}$, and pressure powders of magnetic flux density $B_{20 \mathrm{k}}$ of $1.95 \mathrm{~T}$.

Results confirmed that these conditions provided high resistivity and high magnetic flux density by application to an insulative film of spinel-type ferrite. Actual SEM images showing the surface state (Fig. 9(a)) of the spinel-type ferrite coating powder and a section of the compaction (Fig. 9(b)) are shown in Fig. 9. Most of the coating surface was coated
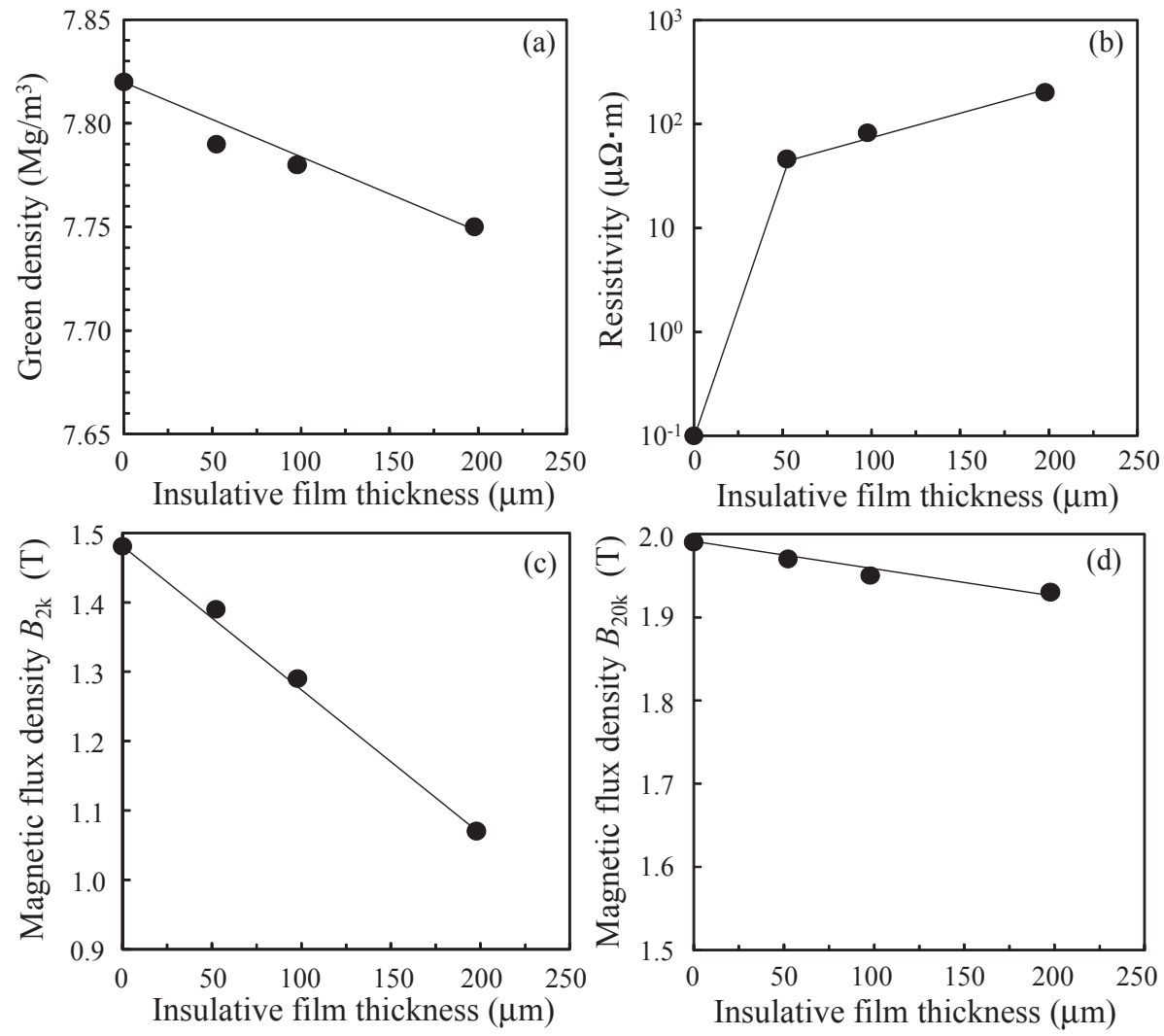

Fig. 8 Effect of insulative film thickness on the properties.
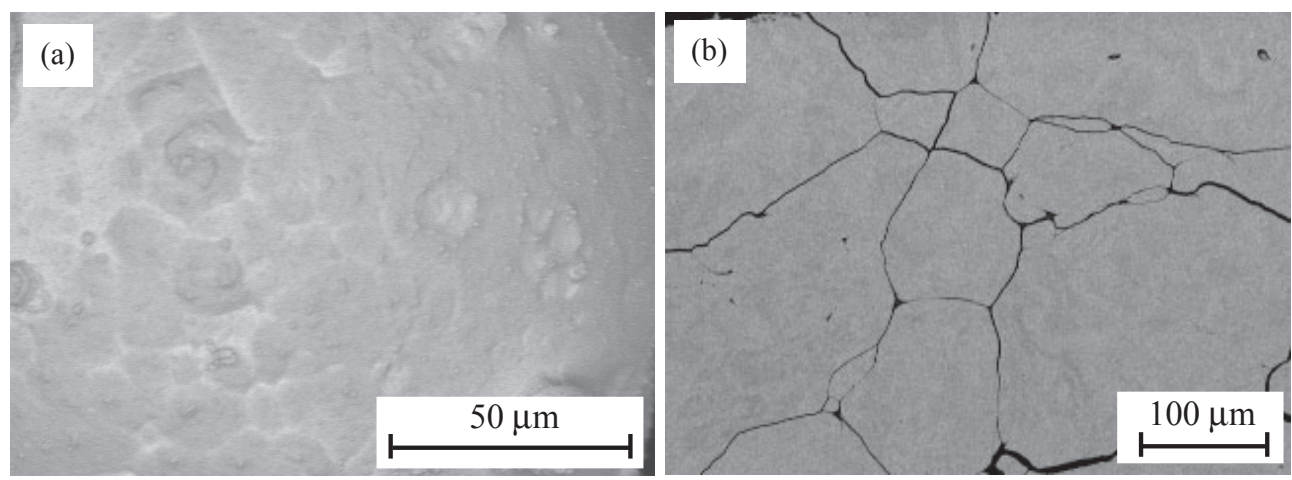

Fig. 9 SEM images of (a) surface of powder coated by spinel-type ferrite and (b) magnetic core cross-section. 


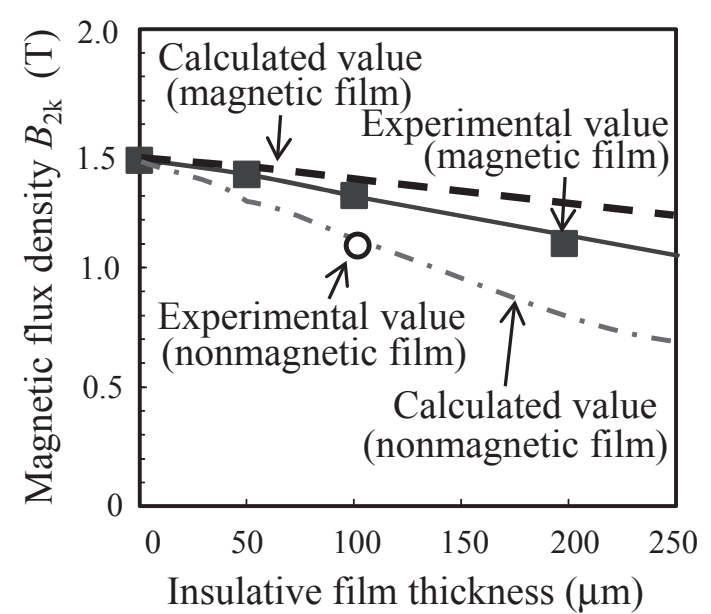

Fig. 10 Comparison of experimental and calculated values (magnetic flux density $\left.B_{2 \mathrm{k}}\right)$.

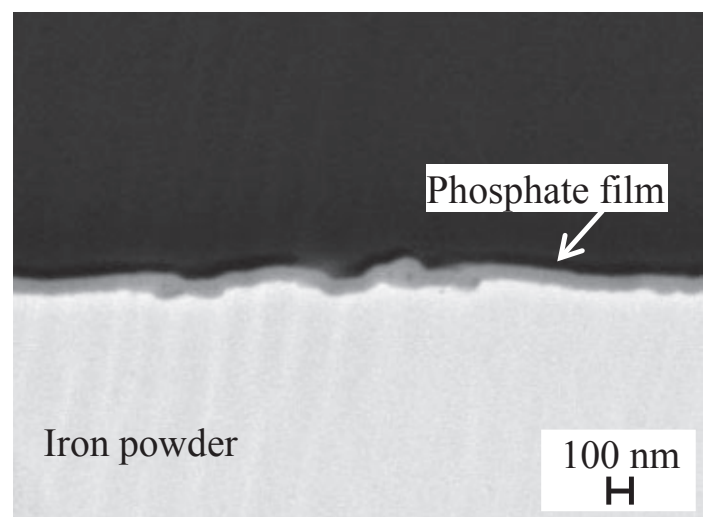

Fig. 11 SEM image of the cross section of powder plated by nonmagnetic film.

uniformly. A dense uniform film was observed from a section image. Even for spinel-type ferrite powders coated by simple mixing, results demonstrate the possibility of coating particles uniformly and achieving compaction with balanced high resistivity with high magnetic flux density.

\subsection{Effect of higher magnetic flux density with the magnetic insulative film}

To compare experimental values with calculated values of magnetic flux density $B_{2 \mathrm{k}}$ of the magnetic and nonmagnetic films, a ring test piece was manufactured using raw magnetic powders and phosphate coating powders at equal compaction pressures of $1568 \mathrm{MPa}$. Experimental and calculated values of magnetic flux density $B_{2 \mathrm{k}}$ in magnetic and nonmagnetic insulative film thicknesses are presented in Fig. 10. The thickness of the phosphate film, a nonmagnetic film, was measured directly from an SEM section image of the coating powder. Results show that the phosphate film thickness was approximately $100 \mathrm{~nm}$. An SEM section image of the phosphate film coating powder is presented in Fig. 11. Magnetic flux density $B_{2 \mathrm{k}}$ of the powder magnetic core having both magnetic and nonmagnetic film decreased as the film thickness increased, but the magnetic flux density $B_{2 \mathrm{k}}$ of the magnetic film was higher than that of the nonmagnetic insulative film.

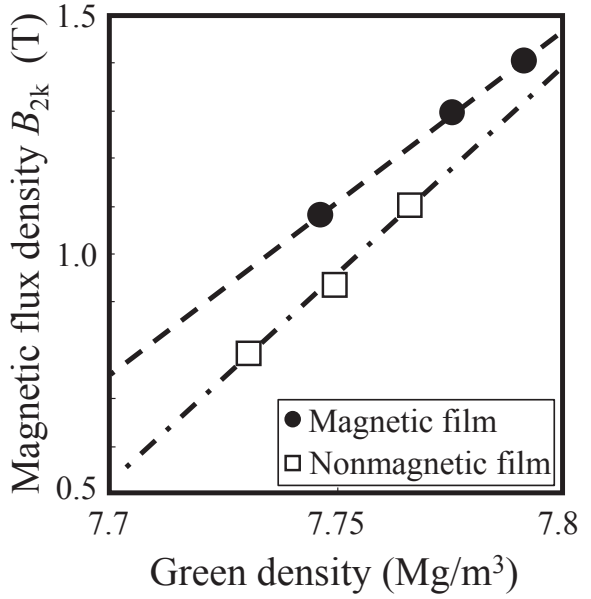

Fig. 12 Comparison of magnetic flux density $B_{2 \mathrm{k}}$ between magnetic film core and nonmagnetic film core.

Table 1 Properties of powder magnetic cores with magnetic insulative film and nonmagnetic insulative film using Fig. 13.

\begin{tabular}{lcccc}
\hline & $\begin{array}{c}\text { Green density } \\
\left(\mathrm{Mg} / \mathrm{m}^{3}\right)\end{array}$ & $\begin{array}{c}\text { Resitivity } \\
(\mu \Omega \cdot \mathrm{m})\end{array}$ & $\begin{array}{c}B_{2 \mathrm{k}} \\
(\mathrm{T})\end{array}$ & $\begin{array}{c}B_{20 \mathrm{k}} \\
(\mathrm{T})\end{array}$ \\
\hline Magnetic film & 7.78 & 100 & 1.29 & 1.95 \\
Nonmagnetic film & 7.77 & 100 & 1.10 & 1.86 \\
\hline
\end{tabular}

Additionally, for experimental values up to $100 \mathrm{~nm}$ thickness, the tendency of magnetic flux density $B_{2 \mathrm{k}}$ to change depending on the magnetic insulative film thickness is almost equal to that found for calculated values. In the nonmagnetic insulative film, the calculated value was well matched with the experimental value at $100 \mathrm{~nm}$ thickness. However, the gap separating the calculated and the experimental value became greater at film thickness of greater than $200 \mathrm{~nm}$, which is almost double the film thickness of the magnetic powder core (400-500 nm).

Presumably, cavities would not be formed other than according to the film thickness, but cavities were actually formed during compaction, so that the green density of magnetic core was decreased. Therefore, the decrease of green density caused by the cavity generation was assumed as the cause of increase of the gap separating the calculated and experimental value at beyond $200 \mathrm{~nm}$ and greater film thickness.

The change of magnetic flux density $B_{2 \mathrm{k}}$, which depends on the green density of a powder magnetic core with both magnetic and a nonmagnetic insulative film, is presented in Fig. 12. Magnetic flux density $B_{2 \mathrm{k}}$ improved along with the increase of green density. The magnetic flux density $B_{2 \mathrm{k}}$ for a magnetic film was higher than that for a nonmagnetic film of equal density.

Table 1 presents evaluation results of the green density of magnetic cores, resistivity, magnetic flux density $B_{2 \mathrm{k}}$ and $B_{20 \mathrm{k}}$ of powder magnetic cores having equal resistivity of $100 \mu \Omega \cdot \mathrm{m}$ with nonmagnetic and magnetic films. The $B-H$ curves of both insulative films are presented in Fig. 13. From these results, it was inferred that the magnetism of the insulative film by the spinel-type ferrite coating enabled 


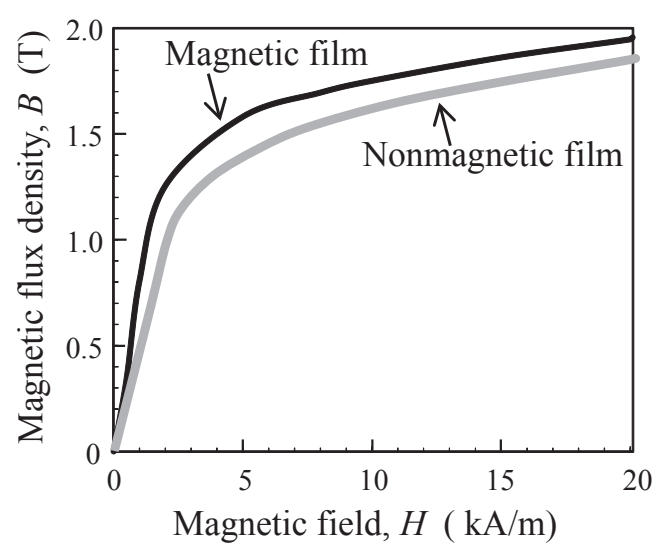

Fig. 13 Initial magnetization curves of magnetic film and nonmagnetic film cores.

higher magnetic flux density of the magnetic powder core while securing insulation characteristics.

\section{Conclusion}

The properties of a powder magnetic core comprising iron powders coated with spinel-type ferrite powders were evaluated to ascertain the high magnetic flux density of the powder magnetic core according to the magnetic insulative film. The following results were clarified.

(1) Evaluation of the magnetic flux density of the powder magnetic cores having spinel-type ferrite insulative film revealed that experimental values matched calculated values. The calculation results show that the effects of magnetic insulative films on raising magnetic flux density were most notable when the film thickness was increased and the powder diameter was increased.

(2) Results demonstrated that the magnetic flux density of powder magnetic core with the magnetic insulative films was higher than that of a nonmagnetic insulative film of the same resistivity. The insulative film achieved higher magnetic flux density of the powder magnetic core.

(3) Results confirmed the possibility of insulation of the powder magnetic core of compacted spinel-type ferrite coated iron powders. Such powder magnetic cores compacted at $1568 \mathrm{MPa}$ have the following properties: $70 \mu \Omega \cdot \mathrm{m}$ resistivity, $7.79 \mathrm{Mg} / \mathrm{m}^{3}$ green density, and magnetic flux density $B_{2 \mathrm{k}}$ of $1.4 \mathrm{~T}$.

\section{REFERENCES}

1) Y. Ozaki and M. Fujinaga: Kawasaki Steel Giho 31 (1999) 130-134.

2) H. Mitani, A. Hanaki, H. Yaguchi and Y. Seki: Kobe Steel Engineering Reports 48 (1998) 25-28.

3) T. Saito and S. Yahagi: Denkiseiko 69 (1998) 181-187.

4) Y. Shimada, K. Matsunuma, T. Maeda, T. Nishioka and A. Ikegaya: J. Jpn. Soc. Powder Powder Metall. 55 (2008) 149-151.

5) K. Kanagawa, K. Mori and R. Nakayama: Abstracts of Autumn Meeting JSPM, (2005) p. 138.

6) Y. Enomoto, M. Ito, R. Masaki and K. Asaka: IEEJ Trans. Ind. Appl. 126 (2006) 1585-1590.

7) D. Kim, M. Ohnishi, N. Matsushita and M. Abe: IEEE Mag. Trans. 39 (2003) 3181-3183.

8) N. Matsushita, D. Kim and M. Abe: IEEE Mag. Trans. 42 (2006) $2824-$ 2826.

9) M. Abe and Y. Tamaura: Jpn. J. Appl. Phys. 22 (1983) L511-L513.

10) M. Abe: J. Mag. Soc. Jpn. 22 (1998) 1225-1232.

11) K. Fujimura, M. Kato and K. Hirota: J. Jpn. Soc. Powder Powder Metall. 55 (2008) 629-636.

12) M. Obata, M. Kato and K. Hirota: J. Jpn. Soc. Powder Powder Metall. 57 (2010) 672.

13) S. Tajima, T. Hattori, S. Hotta, M. Kondoh, H. Okajima, K. Higashiyama and H. Kishimoto: J. Jpn. Soc. Powder Powder Metall. 50 (2003) 577-583.

14) Y. Kawase, T. Yamaguchi, T. Takemoto, K. Hiramoto and H. Nakai: The Papers of Technical Meeting IEEJ, RM-07-98, (2007) pp. 6972.

15) S. Tajima, T. Hattori, M. Kondoh, H. Kishimoto, M. Sugiyama and T. Kikko: J. Jpn. Soc. Powder Powder Metall. 52 (2005) 164-170.

16) M. Kondoh and H. Okajima: Adv. Powder Metall. Particulate Mater. 3 (2002) 47-54.

17) S. Tajima, T. Hattori, M. Kondoh, M. Sugiyama, K. Higashiyama, H. Kishimoto and T. Kikko: Mater. Trans. 45 (2004) 1891-1894. 